\title{
RINGS WITH EVERY PROPER IMAGE \\ A PRINCIPAL IDEAL RING
}

\author{
P. F. SMITH
}

\begin{abstract}
The main result of this paper states that if $\boldsymbol{R}$ is a right Noetherian right bounded prime ring such that nonzero prime ideals are maximal and such that every proper homomorphic image of $R$ is a principal right ideal ring then $R$ is right hereditary.
\end{abstract}

In [10, Theorem 8] it is proved that if $R$ is a right bounded prime ring of finite right Goldie dimension such that every proper homomorphic image is a right Artinian principal right ideal ring then $R$ is right hereditary. It is not difficult to see that such rings are in fact right Noetherian and so [10, Theorem 8 ] is a consequence of the main result of this present paper. In fact, it is shown here that if $R$ is a right Noetherian right bounded prime ring, such that nonzero prime ideals are maximal and such that for all nonzero prime ideals $P, Q$ (not necessarily distinct) the ring $R / P Q$ is a principal right ideal ring, then $R$ is right hereditary (compare [10, Theorem 9]).

In a recent paper, Hajarnavis and Norton [5, Theorem 6.4] proved that if $R$ is a (right and left) Noetherian right bounded prime ring whose proper homomorphic images are ipri-rings then $R$ is a Dedekind prime ring. The proof involves localization at every nonzero prime ideal of the ring $R$. We show how to deduce this result and in so doing completely remove localization techniques from the proof, making it much more elementary.

If $I$ is any ideal of a ring $R$ then $C(I)$ will denote the set of elements $c$ in $R$ such that $c+I$ is a regular element of the ring $R / I$. The ring $R$ is an ipri-ring if every (two-sided) ideal is principal as a right ideal.

LEMMA 1. Let $R$ be a right Noetherian prime ring such that every proper homomorphic image is an ipri-ring. If $M_{1}$ and $M_{2}$ are distinct maximal ideals of $R$ then $M_{1} M_{2}=M_{2} M_{1}=M_{1} \cap M_{2}$.

Proof. Since $R$ is prime, but not simple, it follows that $M_{1} M_{2} \cap M_{2} M_{1} \neq 0$. Let $\bar{R}=R /\left(M_{1} M_{2} \cap M_{2} M_{1}\right)$. Then $\bar{R}$ is a right Noetherian ipri-ring and $\bar{M}_{1}$ and $\bar{M}_{2}$ are distinct maximal ideals of $\bar{R}$, where - denotes images in $\bar{R}$. There exist elements $c_{1}, c_{2}$ in $R$ such that $\bar{M}_{1}=\bar{c}_{1} \bar{R}$ and $\bar{M}_{2}=\bar{c}_{2} \bar{R}$. By [2, Theorem 3.9], $c_{1} \in \mathcal{C}\left(M_{2}\right)$ and $c_{2} \in \mathcal{C}\left(M_{1}\right)$. It follows that $\bar{M}_{1} \cap \bar{M}_{2} \subseteq \bar{c}_{1} \bar{M}_{2}=\bar{M}_{1} \bar{M}_{2}$ and hence $M_{1} \cap M_{2} \subseteq M_{1} M_{2}$. Thus $M_{1} \cap M_{2}=M_{1} M_{2}$ and similarly $M_{1} \cap M_{2}=M_{2} M_{1}$. 
An ideal $I$ of a ring $R$ has the right $A R$ property if for each right ideal $E$ there exists a positive integer $n$ such that $E \cap I^{n} \subseteq E I$.

LEMMA 2 (SEE [7, THEOREM 1.2]). Let $R$ be a right Noetherian prime ring and I a proper ideal such that $I$ has the right $A R$ property. Then $\cap_{n=1}^{\infty} I^{n}=0$.

If $E$ is a right ideal of a ring $R$ then the bound of $E$ is the unique largest (two-sided) ideal contained in $E$. The ring $R$ is right bounded if every essential right ideal has nonzero bound.

Lemma 3. Let $R$ be a right Noetherian right bounded prime ring such that every proper homomorphic image is an ipri-ring. Then the following statements are equivalent.

(i) Nonzero prime ideals of $R$ are maximal ideals.

(ii) Every ideal of $R$ has the right $A R$ property.

Proof. (i) $\Rightarrow$ (ii) By Lemma 1 and [4, Proposition 3.2].

(ii) $\Rightarrow$ (i) Let $P \subseteq Q$ be distinct prime ideals of $R$. Suppose $P \neq 0$. Then the ring $R / P^{2}$ is an ipri-ring. Let $\bar{R}=R / P^{2}$ and let ${ }^{-}$denote images in $\bar{R}$. There exists an element $q$ in $Q$ such that $\bar{Q}=\bar{q} \bar{R}$. By [2, Theorem 3.9] $q \in \mathcal{C}(P)$ and it follows that $\bar{P}=\bar{q} \bar{P}$. Thus $P=q P+P^{2} \subseteq Q P$. Hence $P=Q P=Q^{2} P=\cdots$ and $Q=$ $R$, for otherwise $P \subseteq \cap_{n=1}^{\infty} Q^{n}=0$ by Lemma 2 .

LEMMA 4. Let $R$ be a right Noetherian prime ring and $I$ a proper ideal of $R$ such that $I$ has the right $A R$ property. If $E$ is an essential right ideal of $R$ and $c$ an element of $E$ such that $E=c R+E I$ then $c$ is regular.

Proof. Since $E=c R+E I=c R+E I^{2}=\cdots$ it follows that

$$
E \leqslant \bigcap_{n=1}^{\infty}\left(c R+I^{n}\right) .
$$

By [2, Theorem 3.9] $E$ contains a regular element $e$. Let $F=e R+c R$. There exists a positive integer $m$ such that $F \cap I^{m} \leqslant F I$. There exists $r$ in $R$ such that $e \in c r+I^{m}$, so that $e-c r \in F I$. Thus $e-c r=e a+c b$ for some elements $a, b$ in $I$. Thus $e(1-a)=c s$ where $s=r+b$. By Lemma 2, $1-a$ is regular in $R$ and hence $c s$ is regular in $R$. If $r(s)$ denotes the right annihilator of the element $s$ then $r(s)=0$ and by [2, Lemma 3.8] $s R$ is an essential right ideal of $R$. Let $u \in R$ satisfy $c u=0$. If $u \neq 0$ then there exists $v, w$ in $R$ such that $0 \neq u v=s w$. Then $c u=0$ implies $w=0$, a contradiction. Thus $r(c)=0$ and it follows that $c$ is regular in $R$.

The next lemma is concerned with the question of when a right ideal which contains a projective right ideal is itself projective.

LEMMA 5. Let $E$ be a right ideal of a ring $R$ such that $E$ contains a projective right ideal $P$. If there exists a right ideal $F$ such that $E \cap F \subseteq P$ and $E+F=R$ then $E$ is projective.

Proof. Clearly $R / P=(E / P) \oplus(F+P) / P$. Since $P$ is projective it follows that $R / P$ has projective dimension $\leqslant 1$. Thus $(F+P) / P$ has projective dimension $\leqslant 1$ and, because $R / E \cong(F+P) / P$, so has $R / E$. Thus $E$ is projective. 
THEOREM 6. Let $R$ be a right Noetherian right bounded prime ring such that nonzero prime ideals are maximal and every proper homomorphic image is a principal right ideal ring. Then $R$ is right hereditary.

Proof. By Lemma 3 every ideal of $R$ has the right AR property. Let $E$ be an essential right ideal of $R$ and let $I$ be the bound of $E$. Then $E I$ is an essential right ideal of $R$. Let $A$ be the bound of $E I$. Since $R / A$ is a principal right ideal ring there exists an element $c$ in $E$ such that $E=c R+E I$. By Lemma 4, $c$ is regular. Thus $c R$ is an essential right ideal of $R$ (see [2, Lemma 3.8]). Let $B$ be the bound of $c R$. Then $A+B \subseteq E$. Since $R$ is right Noetherian the nonzero ideal $B$ contains a product of nonzero prime, and hence maximal, ideals, say $M_{1} M_{2} \cdots M_{n} \subseteq B$ with each $M_{i}$ a maximal ideal of $R$. Since $A+B \subseteq E$ it follows that $A \subseteq M_{i}$ for some $1 \leqslant i \leqslant n$ and by Lemma 1 we can suppose without loss of generality that $i=1$. By rearranging the ideals $M_{i}(1 \leqslant i \leqslant n)$ using Lemma 1 , if necessary, we may suppose that there exists a positive integer $m \leqslant n$ such that $I \subseteq M_{i}(1 \leqslant i<m)$ and $I \nsubseteq M_{i}(m+1 \leqslant i \leqslant n)$.

Since $E=c R+E I=c R+E I^{2}=\cdots$ it follows that $E \subseteq c R+I^{m}$. If $m=$ $n$ then $I^{n} \subseteq B \subseteq c R$ and hence $E \subseteq c R$. Thus $E=c R$ and $E$ is projective. Otherwise, if $m<n$ let $J=M_{m+1} \cap \cdots \cap M_{n}$. Then $I^{m} J \subseteq c R$ and hence $E J \subseteq$ $c R$. There exists a positive integer $k$ such that $E \cap J^{k} \subseteq E J \subseteq c R$. Moreover, $I+J=R$ implies that $I+J^{k}=R$ and hence $E+J^{k}=R$. By Lemma 5, $E$ is projective. It follows that every essential right ideal of $R$ is projective. But every right ideal of $R$ is a direct summand of an essential right ideal. Hence $R$ is right hereditary.

In what follows, by a Noetherian ring we shall mean a right and left Noetherian ring. If $I$ is an ideal of a ring $R$ then we call a right ideal $E$ essential modulo $I$ if $I \subseteq E$ and $E / I$ is an essential right ideal of the ring $R / I$. The next result is well known.

Lemma 7. Let $M$ be a maximal ideal of a Noetherian ring $R$. Then $C(M)=$ $\mathcal{C}\left(M^{k}\right)$ for all positive integers $k$.

Proof. Suppose $C(M)=C\left(M^{k}\right)$ for some $k \geqslant 1$. We shall prove that $C(M)=$ $\mathcal{C}\left(M^{k+1}\right)$. The result will then follow by induction. By a result of Djabali (see [3, Theorem 2.1]), $\mathcal{C}\left(M^{k+1}\right) \subseteq \mathcal{C}(M)$. Let

$$
K=\left\{r \in R: r c \in M^{k+1} \text { for some } c \text { in } \mathcal{C}(M)\right\} .
$$

Then $K \leqslant M^{k}$. By [2, Theorem 3.9] a right ideal $E$ is essential modulo $M$ if and only if $c R+M \subseteq E$ for some element $c$ in $C(M)$. Thus

$$
K=\left\{r \in R: r E \subseteq M^{k+1} \text { for some right ideal } E \text { essential modulo } M\right\} .
$$

By the properties of essential right ideals of $R / M$ it follows that $K$ is an ideal of $R$. Since $R$ is left Noetherian the left ideal $K$ is finitely generated and hence $K d \subseteq M^{k+1}$ for some $d$ in $\bigodot(M)$. But $K M \subseteq M^{k+1}$ and thus

$$
M \varsubsetneqq\left\{r \in R: K r \subseteq M^{k+1}\right\}=L .
$$


Because $M$ is maximal, $L=R$ and hence $K \subseteq M^{k+1}$. Thus whenever $r \in R$, $c \in \mathcal{C}(M)$ and $r c \in M^{k+1}$ we have $r \in M^{k+1}$. By a similar argument it can be shown that if $c r \in M^{k+1}$ with $r$ in $R$ and $c$ in $C(M)$ then $r \in M^{k+1}$. Thus $\mathcal{C}(\boldsymbol{M}) \subseteq \mathcal{C}\left(\boldsymbol{M}^{k+1}\right)$ and we conclude $\mathcal{C}(\boldsymbol{M})=\mathcal{C}\left(\boldsymbol{M}^{k+1}\right)$.

LEMMA 8 (SEE [6, LEMMA 3.1]). Let $I=a R$ be an ideal of $a$ Noetherian ring $R$ such that $I \subseteq \cap_{n=1}^{\infty} b^{n} R$ for some element $b$ in $R$. Then there exists $c$ in $R$ such that $(1-c b) I=0$.

LEMMA 9. Let $R$ be a Noetherian right bounded prime ring such that every proper homomorphic image is an ipri-ring. If $P$ is a nonzero prime ideal of $R$ then the ring $R / P$ is right Artinian.

Proof. Let $Q \subseteq M$ be distinct prime ideals of $R$ and suppose $Q \neq 0$. Then the ring $R / Q^{2}$ is an ipri-ring. Let $\bar{R}=R / Q^{2}$ and ${ }^{-}$denote images in $\bar{R}$. As in the proof of Lemma 3 (ii) $\Rightarrow$ (i), $\bar{Q}=\bar{M} \bar{Q}$ and hence $\bar{Q} \subseteq \cap_{n=1}^{\infty} \bar{M}^{n}$. By Lemma 8, there exists an element $m$ in $M$ such that $(\overline{1}-\bar{m}) \bar{Q}=\overline{0}$. By [6, Corollary 3.4], $\overline{1}-\bar{m}$ is nilpotent and hence $M=R$. Thus nonzero prime ideals of $R$ are maximal. Also by Lemma 1 maximal ideals of $R$ commute.

Let $P$ be a nonzero prime ideal of $R$. Let $c \in \mathcal{C}(P)$. By Lemma $3 P$ has the right AR property and by Lemma $2 \cap_{n=1}^{\infty} P^{n}=0$. Hence by Lemma 7,c $\in \mathcal{C}(0)$. Thus $c R$ is an essential right ideal of $R$ (see [2, Theorem 3.9]) and there exist maximal ideals $M_{1}, \ldots, M_{k}$ for some positive integer $k$ such that $M_{1} M_{2} \cdots M_{k} \subseteq c R$. Suppose that $P \neq M_{i}(1 \leqslant i \leqslant k)$. Then there exists an element $p$ in $P$ such that $1-p \in M_{1} M_{2} \cdots M_{k} \subseteq c R$. It follows that $c+P$ is a unit in the ring $R / P$. Therefore, suppose $P=M_{i}$ for some $1 \leqslant i<k$. Then there exists a positive integer $t$ and an element $x$ in $P$ such that $P^{t}(1-x) \subseteq c R$. In this case, $c \in \mathcal{C}(P)$ implies

$$
P^{t}=c P^{t}+P^{t+1}
$$

by Lemma 7. Let $R^{*}=R / P^{t+1}$ and let ${ }^{*}$ denote images in $R^{*}$. Since $R^{*}$ is an ipri-ring it follows that $\left(P^{t}\right)^{*}$ is principal as a right ideal and

$$
\left(P^{t}\right)^{*} \subseteq \bigcap_{n=1}^{\infty} c^{* n} R^{*} .
$$

By Lemma 8 there exists an element $a$ in $R$ such that

$$
\left(1^{*}-a^{*} c^{*}\right)\left(P^{t}\right)^{*}=0 .
$$

Thus $(1-a c) P^{t} \subseteq P^{t+1}$. Let $K=\left\{r \in R: r P^{t} \subseteq P^{t+1}\right\}$. Then $K$ is an ideal of $R$ and $P \subseteq K$. If $K=R$ then $P^{t}=P^{t+1}$ and so $P^{t}=0$ by Lemma 2. Thus $K=P$ and hence $1-a c \in P$. It follows that $c+P$ is a unit in $R / P$. Thus, for each element $c$ in $C(P)$ we have proved that $c+P$ is a unit in $R / P$. By [2, Theorems 4.1 and 4.4] the ring $R / P$ is right Artinian.

Given Lemma 9, the next result is well known but we include it and its proof for completeness.

TheOREM 10. Let $R$ be a Noetherian right bounded prime ring such that every proper homomorphic image is an ipri-ring. Then $R$ is a Dedekind prime ring. 
Proof. Let $I$ be a nonzero ideal of $R$. There exist a positive integer $k$ and prime ideals $P_{1}, \ldots, P_{k}$ such that $P_{1} \cdots P_{k} \subseteq I$. By Lemma 9 the ring $R / I$ is right Artinian. In addition $R / I$ is an ipri-ring and hence $R / I$ is a principal right ideal ring by [6, Theorem 2.8]. Thus every proper homomorphic image of $R$ is a principal right ideal ring and every nonzero prime ideal is maximal. By Theorem $6, R$ is right hereditary. Since $R$ is left Noetherian it follows that $R$ is left hereditary and by combining Lemmas 2 and 3 with [1, Theorem 1.2] we see that $R$ is a Dedekind prime ring.

LemMa 11. Let $R$ be a Noetherian prime ring such that the ring $R / P Q$ is an ipri-ring for all nonzero prime ideals $P, Q$. Then the ring $R / I$ is an ipri-ring for all nonzero ideals $I$ of $R$.

Proof. Note first that nonzero prime ideals of $R$ are maximal ideals. Let $P \subseteq M$ be distinct prime ideals of $R$ with $P \neq 0$. Then $R / P^{2}$ is an ipri-ring. Let $\bar{R}=$ $R / P^{2}$ and - denote images in $\bar{R}$. Then $\bar{P}=\bar{M} \bar{P}$ by the proof of Lemma 3 (ii) $\Rightarrow$ (i). Thus $\bar{P} \subseteq \cap_{n=1}^{\infty} \bar{M}^{n}$ and by Lemma 8 there exists an element $m$ in $\bar{M}$ such that $(1-\bar{m}) \bar{P}=0$. By [6, Corollary 3.4], $1-\bar{m}$ is nilpotent and hence $M=R$.

Now let $P$ and $Q$ be distinct maximal ideals of $R$. By hypothesis the ring $R^{*}=R / P Q$ is an ipri-ring. If ${ }^{*}$ denotes images in $R^{*}$ then $P^{*}=p^{*} R^{*}$ for some element $p$ in $R$. Since $P \nsubseteq Q$, it follows that $p \in \mathcal{C}(Q)$ (see [2, Theorems 3.9]). Hence $P^{*} \cap Q^{*} \subseteq p^{*} Q^{*}=0$. Thus $P \cap Q \subseteq P Q$ and this gives $P \cap Q=P Q$. Similarly by considering the ring $R / Q P$ we have $P \cap Q=Q P$. Thus nonzero prime ideals of $R$ commute.

Let $I$ be a nonzero ideal of $R$. Then there exist positive integers $k, n_{1}, \ldots, n_{k}$ and distinct maximal ideals $M_{1}, \ldots, M_{k}$ such that

$$
M_{1}^{n_{1}} M_{2}^{n_{2}} \cdots M_{k}^{n_{k}} \subseteq I .
$$

By the Chinese Remainder Theorem

$$
R /\left(M_{1}^{n_{1}} \cdots M_{k}^{n_{k}}\right) \cong \bigoplus_{i=1}^{k}\left(R / M_{i}^{n_{i}}\right)
$$

Moreover, by [5, Theorem 4.1] the ring $R / M_{i}^{n_{i}}$ is an ipri-ring for each $1<i<k$. It follows that the ring $R /\left(M_{1}^{n_{1}} \cdots M_{k}^{n_{k}}\right)$, and hence the ring $R / I$, is an ipri-ring.

Combining Lemma 11 with Theorems 6 and 10 we have as our final result

COROLlary 12. Let $R$ be a right Noetherian right bounded prime ring such that either (a) nonzero prime ideals of $R$ are maximal and $R / P Q$ is a principal right ideal ring for all nonzero prime ideals $P$ and $Q$ of $R$, or (b) $R$ is left Noetherian and $R / P Q$ is an ipri-ring for all nonzero prime ideals $P$ and $Q$ of $R$. Then $R$ is right hereditary.

[9] gives an example of a Noetherian simple ring $S$ which has infinite global dimension but Krull dimension one. It is shown in [8] that if $E$ is an essential right ideal of $S$ and $c$ any regular element in $E$ then the right $S$-module $E / c S$ is cyclic. This example highlights the fact that Theorem 6 is a result about right bounded rings. 


\section{REFERENCES}

1. D. Eisenbud and J. C. Robson, Hereditary Noetherian prime rings, J. Algebra 16 (1970), 86-104.

2. A. W. Goldie, Semiprime rings with maximum condition, Proc. London Math. Soc. (3) 10 (1960), 201-220.

3. Some aspects of ring theory, Bull London Math. Soc. 1 (1969), 129-154.

4. C. R. Hajarnavis, Non-commutative rings whose homomorphic images are self-injective, Bull. London Math. Soc. 5 (1973), 70-74.

5. C. R. Hajarnavis and N. C. Norton, The one and half generator property in Noetherian rings, University of Warwick, February 1979, preprint.

6. J. C. Robson, Pri-rings and ipri-rings, Quart J. Math. Oxford Ser. (2) 18 (1967), 125-145.

7. P. F. Smith, On the intersection theorem, Proc. London Math. Soc. (3) 21 (1970), 385-398.

8. J. T. Stafford, Completely faithful modules and ideals of simple Noetherian rings, Bull. London Math. Soc. 8 (1976), 168-173.

9. (1978), 159-160.

10. A. Zaks, Some rings are hereditary rings, Israel J. Math. 10 (1971), 442-450.

Department of Mathematics, University of Glasgow, Glasgow G12 8QW, Scotland 